\title{
A Compact Formula for Rotations as Spin Matrix Polynomials
}

Thomas L. CURTRIGHT ${ }^{\dagger}$, David B. FAIRLIE $\ddagger$ and Cosmas K. ZACHOS $\S$

$\dagger$ Department of Physics, University of Miami, Coral Gables, FL 33124-8046, USA

E-mail: curtright@miami.edu

URL: http://www.physics.miami.edu/ curtright/

$\ddagger$ Department of Mathematical Sciences, Durham University, Durham, DH1 3LE, UK

E-mail: David.Fairlie@durham.ac.uk

$\S$ High Energy Physics Division, Argonne National Laboratory, Argonne, IL 60439-4815, USA

E-mail: c.zachos.k@att.net

URL: http://www.hep.anl.gov/czachos/

Received May 07, 2014, in final form August 07, 2014; Published online August 12, 2014

http://dx.doi.org/10.3842/SIGMA.2014.084

\begin{abstract}
Group elements of $\mathrm{SU}(2)$ are expressed in closed form as finite polynomials of the Lie algebra generators, for all definite spin representations of the rotation group. The simple explicit result exhibits connections between group theory, combinatorics, and Fourier analysis, especially in the large spin limit. Salient intuitive features of the formula are illustrated and discussed.
\end{abstract}

Key words: spin matrices; matrix exponentials

2010 Mathematics Subject Classification: 15A16; 15A30

\section{Introduction}

Rotation matrices, worked out by Wigner [15] for all representations, are one of the mainstays of quantum mechanics. They amount to group elements of $\mathrm{SU}(2)$, exponentials $e^{i \theta(\hat{\boldsymbol{n}} \cdot \boldsymbol{J})}$ of suitable Lie algebra $\left(\left[J_{a}, J_{b}\right]=i \epsilon_{a b c} J_{c}\right)$ generators. Of course, these are $(2 j+1) \times(2 j+1)$ dimensional matrices for either integral spin, $j=0,1,2,3, \ldots$ or half-integral spin, $j=\frac{1}{2}, \frac{3}{2}, \frac{5}{2}, \ldots$ Here, $\theta$ is the rotation angle around the unit vector axis $\hat{\boldsymbol{n}}$, and the third component of the matrix triplet $\boldsymbol{J}$ in standard convention is the diagonal matrix $J_{3}=\operatorname{diag}(j, j-1, j-2, \ldots, 1-j,-j)$.

By the Cayley-Hamilton theorem, the expansion of this exponential in powers of $\hat{\boldsymbol{n}} \cdot \boldsymbol{J}$ may be recast as a finite sum of powers of $\hat{\boldsymbol{n}} \cdot \boldsymbol{J}$, the highest power being of order $2 j$. Such polynomials of Lie generators, in the universal enveloping algebra of $s u(2)$, have numerous celebrated applications in physics.

Nevertheless, beyond standard expressions for $j=1 / 2$, so $\boldsymbol{J}=\boldsymbol{\sigma} / 2$,

$$
e^{i(\theta / 2)(\hat{\boldsymbol{n}} \cdot \boldsymbol{\sigma})}=I_{2} \cos \theta / 2+i(\hat{\boldsymbol{n}} \cdot \boldsymbol{\sigma}) \sin \theta / 2,
$$

and the triplet, $j=1$, so $J_{3}=\operatorname{diag}(1,0,-1)$,

$$
\begin{aligned}
e^{i \theta(\hat{\boldsymbol{n}} \cdot \boldsymbol{J})} & =I_{3}+i(\hat{\boldsymbol{n}} \cdot \boldsymbol{J}) \sin \theta+(\hat{\boldsymbol{n}} \cdot \boldsymbol{J})^{2}(\cos \theta-1) \\
& =I_{3}+(2 i \hat{\boldsymbol{n}} \cdot \boldsymbol{J} \sin (\theta / 2)) \cos (\theta / 2)+\frac{1}{2}(2 i \hat{\boldsymbol{n}} \cdot \boldsymbol{J} \sin (\theta / 2))^{2},
\end{aligned}
$$

which actually amounts to the vector Rodrigues' rotation formula $[4,10]$, such expansions for higher dimensionality multiplets are relatively rare. 
While van Wageningen [13] and Lehrer-Ilamed [5] made substantial progress and provided some significant results (also see the less direct algorithms in [14] and [12]), until now there has not appeared a simple, compact, closed expression illuminating the properties of the expansion for arbitrary $j$. Our methods and spirit clearly overlap those of ref [13], although, crucially, our basic variable is $\sin (\theta / 2)$ and not trigonometric functions of $n \theta$ as in this and other treatments. Because of this, we are able to furnish the ultimate solution of all the algorithms appearing previously in the literature.

\section{New compact results}

A formula valid for arbitrary spin $j$ is given by

$$
e^{i \theta(\hat{\boldsymbol{n}} \cdot \boldsymbol{J})}=\sum_{k=0}^{2 j} \frac{c_{k}(\theta)}{k !}(2 i \hat{\boldsymbol{n}} \cdot \boldsymbol{J} \sin (\theta / 2))^{k},
$$

where, with use of the floor function,

$$
c_{k}(\theta)=(\cos (\theta / 2))^{\epsilon} \operatorname{Trunc}_{\lfloor j-k / 2\rfloor}\left(\frac{(\arcsin \sqrt{x} / \sqrt{x})^{k}}{(\sqrt{1-x})^{\epsilon}}\right)
$$

with $x=\sin ^{2}(\theta / 2)$ and

$$
\epsilon(j-k / 2)=2 j-k-2\lfloor j-k / 2\rfloor=\frac{1-(-1)^{2 j-k}}{2},
$$

a binary "parity" variable: 0 for even $2 j-k$, and 1 for odd $2 j-k$. The effective variable $2 j-k$ is the descending order of this polynomial (1). Trunc ${ }_{n}$ is a Taylor polynomial in $x$, i.e., it denotes truncating the infinite series of its arguments to $O\left(x^{n}\right)$,

$$
\underset{n}{\operatorname{Trunc}}\left(\sum_{m=0}^{\infty} a_{m} x^{m}\right) \equiv \sum_{m=0}^{n} a_{m} x^{m} .
$$

Each term in the Taylor polynomials Trunc $_{n}$ of (2) is positive semi-definite.

The compact formula (1) and its essential features are exemplified and analyzed in the sections to follow. Examples are given in Section 3. Reformulated in descending order, the polynomial coefficients are discussed and inter-related in Section 4. In Section 5, these coefficients are organized into compact generating functions, linking different values of $j$. The machinery needed to derive the results is presented in Section 6. Concluding commentary with special attention to the large spin limit is provided in Section 7, and illustrated in an appendix.

\section{Basic examples and symmetries}

A few low spin $j$ cases of (1) are provided to exemplify the structure of the series.

For the quartet, $j=3 / 2$,

$$
\begin{aligned}
e^{i \theta(\hat{\boldsymbol{n}} \cdot \boldsymbol{J})}= & I_{4} \cos (\theta / 2)\left(1+\frac{1}{2} \sin ^{2}(\theta / 2)\right)+(2 i \hat{\boldsymbol{n}} \cdot \boldsymbol{J} \sin (\theta / 2))\left(1+\frac{1}{6} \sin ^{2}(\theta / 2)\right) \\
& +\frac{1}{2 !}(2 i \hat{\boldsymbol{n}} \cdot \boldsymbol{J} \sin (\theta / 2))^{2} \cos (\theta / 2)+\frac{1}{3 !}(2 i \hat{\boldsymbol{n}} \cdot \boldsymbol{J} \sin (\theta / 2))^{3} .
\end{aligned}
$$

For the quintet, $j=2$,

$$
e^{i \theta(\hat{\boldsymbol{n}} \cdot \boldsymbol{J})}=I_{5}+(2 i \hat{\boldsymbol{n}} \cdot \boldsymbol{J} \sin (\theta / 2)) \cos (\theta / 2)\left(1+\frac{2}{3} \sin ^{2}(\theta / 2)\right)
$$




$$
\begin{aligned}
& +\frac{1}{2 !}(2 i \hat{\boldsymbol{n}} \cdot \boldsymbol{J} \sin (\theta / 2))^{2}\left(1+\frac{1}{3} \sin ^{2}(\theta / 2)\right) \\
& +\frac{1}{3 !}(2 i \hat{\boldsymbol{n}} \cdot \boldsymbol{J} \sin (\theta / 2))^{3} \cos (\theta / 2)+\frac{1}{4 !}(2 i \hat{\boldsymbol{n}} \cdot \boldsymbol{J} \sin (\theta / 2))^{4} .
\end{aligned}
$$

For the sextet, $j=5 / 2$,

$$
\begin{aligned}
e^{i \theta(\hat{\boldsymbol{n}} \cdot \boldsymbol{J})}= & I_{6} \cos (\theta / 2)\left(1+\frac{1}{2} \sin ^{2}\left(\theta / 2+\frac{3}{8} \sin ^{4}(\theta / 2)\right)\right. \\
& +(2 i \hat{\boldsymbol{n}} \cdot \boldsymbol{J} \sin (\theta / 2))\left(1+\frac{1}{6} \sin ^{2}(\theta / 2)+\frac{3}{40} \sin ^{4}(\theta / 2)\right) \\
& +\frac{1}{2 !}(2 i \hat{\boldsymbol{n}} \cdot \boldsymbol{J} \sin (\theta / 2))^{2} \cos (\theta / 2)\left(1+\frac{5}{6} \sin ^{2}(\theta / 2)\right) \\
& +\frac{1}{3 !}(2 i \hat{\boldsymbol{n}} \cdot \boldsymbol{J} \sin (\theta / 2))^{3}\left(1+\frac{1}{2} \sin ^{2}(\theta / 2)\right) \\
& +\frac{1}{4 !}(2 i \hat{\boldsymbol{n}} \cdot \boldsymbol{J} \sin (\theta / 2))^{4} \cos (\theta / 2)+\frac{1}{5 !}(2 i \hat{\boldsymbol{n}} \cdot \boldsymbol{J} \sin (\theta / 2))^{5} .
\end{aligned}
$$

For $\operatorname{spin} j=5$,

$$
\begin{aligned}
e^{i \theta(\hat{\boldsymbol{n}} \cdot \boldsymbol{J})}= & I_{11}+(2 i \hat{\boldsymbol{n}} \cdot \boldsymbol{J} \sin (\theta / 2)) \cos (\theta / 2) \\
& \times\left(1+\frac{2}{3} \sin ^{2}(\theta / 2)+\frac{8}{15} \sin ^{4}(\theta / 2)+\frac{16}{35} \sin ^{6}(\theta / 2)+\frac{128}{315} \sin ^{8}(\theta / 2)\right) \\
& +\frac{1}{2 !}(2 i \hat{\boldsymbol{n}} \cdot \boldsymbol{J} \sin (\theta / 2))^{2} \\
& \times\left(1+\frac{1}{3} \sin ^{2}(\theta / 2)+\frac{8}{45} \sin ^{4}(\theta / 2)+\frac{4}{35} \sin ^{6}(\theta / 2)+\frac{128}{1575} \sin ^{8}(\theta / 2)\right) \\
& +\frac{1}{3 !}(2 i \hat{\boldsymbol{n}} \cdot \boldsymbol{J} \sin (\theta / 2))^{3} \cos (\theta / 2)\left(1+\sin ^{2}(\theta / 2)+\frac{14}{15} \sin ^{4}(\theta / 2)+\frac{164}{189} \sin ^{6}(\theta / 2)\right) \\
& +\frac{1}{4 !}(2 i \hat{\boldsymbol{n}} \cdot \boldsymbol{J} \sin (\theta / 2))^{4}\left(1+\frac{2}{3} \sin ^{2}(\theta / 2)+\frac{7}{15} \sin ^{4}(\theta / 2)+\frac{328}{945} \sin ^{6}(\theta / 2)\right) \\
& +\frac{1}{5 !}(2 i \hat{\boldsymbol{n}} \cdot \boldsymbol{J} \sin (\theta / 2))^{5} \cos (\theta / 2)\left(1+\frac{4}{3} \sin ^{2}(\theta / 2)+\frac{13}{9} \sin ^{4}(\theta / 2)\right) \\
& +\frac{1}{6 !}(2 i \hat{\boldsymbol{n}} \cdot \boldsymbol{J} \sin (\theta / 2))^{6}\left(1+\sin ^{2}(\theta / 2)+\frac{13}{15} \sin ^{4}(\theta / 2)\right) \\
& +\frac{1}{7 !}(2 i \hat{\boldsymbol{n}} \cdot \boldsymbol{J} \sin (\theta / 2))^{7} \cos (\theta / 2)\left(1+\frac{5}{3} \sin ^{2}(\theta / 2)\right) \\
& +\frac{1}{8 !}(2 i \hat{\boldsymbol{n}} \cdot \boldsymbol{J} \sin (\theta / 2))^{8}\left(1+\frac{4}{3} \sin ^{2}(\theta / 2)\right) \\
& +\frac{1}{9 !}(2 i \hat{\boldsymbol{n}} \cdot \boldsymbol{J} \sin (\theta / 2))^{9} \cos (\theta / 2) \\
& +\frac{1}{10 !}(2 i \hat{\boldsymbol{n}} \cdot \boldsymbol{J} \sin (\theta / 2))^{10},
\end{aligned}
$$

etc.

The trace of each such exponential $(2 j+1) \times(2 j+1)$ matrix is the character of this spin $j$ representation of $\mathrm{SU}(2)$, [11, formulas (2.32)-(2.38)], the Gegenbauer and also the 2nd kind Chebyshev polynomial, $C_{2 j}^{1}(\cos (\theta / 2))=U_{2 j}(\cos (\theta / 2))=\sin ((2 j+1) \theta / 2) / \sin (\theta / 2)$. One might note that the above expansions may be expressed as linear combinations of Chebyshev polynomials, as well. Thus, the coefficient of $(\hat{\boldsymbol{n}} \cdot \boldsymbol{J})^{k}$, for integer (semi-integer) $j$ is, respectively, $\quad \sum_{n \leq 2 j} a_{n} T_{n}(\cos (\theta / 2))$ for $k$ even, and $\sum_{n \leq 2 j} b_{n} V_{n}(\cos (\theta / 2))$ for $k$ odd, where $T_{n}(\cos (\theta / 2))=\cos (n \theta / 2)$ (Chebyshev 1st kind) and $V_{n}(\cos (\theta / 2))=\sin (n \theta / 2)=$ $\sin (\theta / 2) U_{n}(\cos (\theta / 2))$ (Chebyshev 2nd kind).

It is evident by taking $k$ derivatives of (1) with respect to $\theta$ and evaluating at $\theta=0$ that the $(i \hat{\boldsymbol{n}} \cdot \boldsymbol{J})^{k}$ term in the series is selected with unit coefficient - all other terms must vanish. It is also necessary, as manifest above, that the last term is of order $2 j$.

For a given $j$, the matrix coefficients of the terms of $\sin ^{2 j}(\theta / 2)$ and $\cos (\theta / 2) \sin ^{2 j-1}(\theta / 2)$ in the expansion, polynomials of order $(\hat{\boldsymbol{n}} \cdot \boldsymbol{J})^{2 j}$ and $(\hat{\boldsymbol{n}} \cdot \boldsymbol{J})^{2 j-1}$ respectively, must vanish for all eigenvalues of $J_{3}$ except the extremal ones, $\pm j$. That is, they are proportional to the characteristic polynomial of $J_{3}[j-1]$ and hence of $\hat{\boldsymbol{n}} \cdot \boldsymbol{J}[j-1]$. Constraining these coefficients 
to zero, which amounts to projecting out these extremal eigenvalues, necessarily reduces the expansion of $e^{i \theta(\hat{\boldsymbol{n}} \cdot \boldsymbol{J}[j])}$ to that of $e^{i \theta(\hat{\boldsymbol{n}} \cdot \boldsymbol{J}[j-1])}$. The reverse procedure, recursive construction of $\exp (i \theta(\hat{\boldsymbol{n}} \cdot \boldsymbol{J}[j]))$ out of $\exp (i \theta(\hat{\boldsymbol{n}} \cdot \boldsymbol{J}[j-1]))$ likewise follows.

For example, the spin $j=3 / 2$ expression leads to the spin $j=5 / 2$,

$$
\begin{aligned}
e^{i \theta(\hat{\boldsymbol{n}} \cdot \boldsymbol{J}[5 / 2])}= & \left.e^{i \theta(\hat{\boldsymbol{n}} \cdot \boldsymbol{J}[3 / 2])}\right|_{[5 / 2]}+\left[\frac{1}{5 !}(2 i \sin (\theta / 2))^{5} \hat{\boldsymbol{n}} \cdot \boldsymbol{J}[5 / 2]+\frac{1}{4 !} \cos (\theta / 2)(2 i \sin (\theta / 2))^{4}\right] \\
& \times\left[\left((\hat{\boldsymbol{n}} \cdot \boldsymbol{J}[5 / 2])^{2}-\left(\frac{3}{2}\right)^{2}\right)\left((\hat{\boldsymbol{n}} \cdot \boldsymbol{J}[5 / 2])^{2}-\left(\frac{1}{2}\right)^{2}\right)\right],
\end{aligned}
$$

where the first term on the right hand side means the polynomial of (3) evaluated for the spin $j=5 / 2,6 \times 6$ matrices, instead of the $4 \times 4$ ones.

All terms, for integral $j$ are actually periodic functions $\theta$, i.e. they can be recast in trigonometric functions of $\theta$, and are thus periodic in $2 \pi$. By contrast, for half-integral spin $j$, all coefficients cannot (they are only trigonometric functions of $\theta / 2$ ), and and thus have period $4 \pi$, instead.

Note the alternating binary parities $\epsilon$, even $(0)$, or odd $(1-$ which inserts a factor $\cos (\theta / 2)$ in the coefficients and effectively in the denominator of the truncated series to be discussed), interleave for a given $j$; and their location shifts by one for a given $k$, going from integral to half-integral spins.

Thus, the odd $\epsilon=1$ series for $k=0$, so then for half-integral spins, truncated to $\lfloor j\rfloor=j-1 / 2$ terms past its leading term 1 is

$$
\begin{aligned}
c_{0}(\epsilon=1) / \cos (\theta / 2) & =1+\frac{1}{2} x+\frac{3}{8} x^{2}+\frac{5}{16} x^{3}+\cdots+\left(\begin{array}{c}
2 j-1 \\
j-1 / 2
\end{array}\right)\left(\frac{x}{4}\right)^{j-1 / 2} \\
& =\frac{1}{\sqrt{\pi}} \sum_{n=0}^{j-\frac{1}{2}} \frac{\Gamma\left(n+\frac{1}{2}\right)}{\Gamma(n+1)} x^{n} .
\end{aligned}
$$

Note the contrast to the likewise odd $\epsilon=1$ series for $k=1$, thus integral spins, truncated to $\lfloor j-1 / 2\rfloor=j-1$ terms past its leading term,

$$
\begin{aligned}
c_{1}(\epsilon=1) / \cos (\theta / 2) & =1+\frac{2}{3} x+\frac{8}{15} x^{2}+\frac{16}{35} x^{3}+\cdots+\frac{(j-1) !(j-1) !}{(2 j-1) !}(4 x)^{j-1} \\
& =\sqrt{\pi} \sum_{n=0}^{j-1} \frac{\Gamma(n+1)}{(2 n+1) \Gamma\left(n+\frac{1}{2}\right)} x^{n} .
\end{aligned}
$$

When the large $j$ limit is considered, the resulting expression would be expected to resemble the expansion of a scalar exponential, as the Cayley-Hamilton theorem applied to higher-order terms provides dwindling corrections - provided the requisite periodicities are respected! The leading coefficient (to the identity) of the expansion, $c_{0}$, is always just 1 , for even $\epsilon$, so then for all integral spins, large and small.

In striking contrast, for odd $\epsilon$, large half-integral spins $j$, the leading term tends to

$$
I_{2 j+1} \operatorname{sgn}(\cos (\theta / 2)) \text {, }
$$

a square waveform with the required periodicity of $4 \pi$. It agrees with the integral spin in $[-\pi, \pi]$, but flips sign outside this interval. (See the first graph in the appendix.)

Similarly, the second term in the expansion linear in the Lie algebra generators, for large integral spins has odd bimodal parity $\epsilon$ and tends to

$$
i \hat{\boldsymbol{n}} \cdot \boldsymbol{J}\left(\theta-2 \pi\left\lfloor\frac{\theta}{2 \pi}-\frac{1}{2}\right\rfloor\right),
$$

a sawtooth forced to maintain periodicity in $2 \pi$. (See the second graph in the appendix.) 
By contrast, for large half-integral spins, even bimodal parity, the limiting triangular waveform can be more symmetric, as the slope of the linear function must reverse at the boundary of $[-\pi, \pi]$,

$$
i \hat{\boldsymbol{n}} \cdot \boldsymbol{J}_{\operatorname{sgn}}(\cos (\theta / 2))\left(\theta-2 \pi\left\lfloor\frac{\theta}{2 \pi}-\frac{1}{2}\right\rfloor\right) .
$$

Thus, for large $j$, all coefficients tend to trigonometric series representations $[9,17]$ as discussed in the last section and illustrated in the appendix.

\section{Equivalent, top-down parameterization of the series of coefficients}

The expansion introduced, (1), may be re-expressed in a descending powers' sum, with the powers of sine now incorporated in the coefficients, $C_{k}[j]=c_{k}(i \sin (\theta / 2))^{k} / k$ !, for convenience,

$$
e^{i \theta(\hat{\boldsymbol{n}} \cdot \boldsymbol{J}[j])}=\sum_{m=0}^{2 j} C_{2 j-m}[j](2 \hat{\boldsymbol{n}} \cdot \boldsymbol{J}[j])^{2 j-m} .
$$

The representation index $[j]$ is also displayed explicitly here since, in the next section, different spins can enter in the same formula; normally this argument may be suppressed when working within a representation, as has been the case so far.

The top-down coefficients are analytic functions of $j$ and apply to either integer or semiinteger spin,

$$
\begin{aligned}
C_{2 j}[j]= & \frac{1}{(2 j) !}(i \sin (\theta / 2))^{2 j}, \\
C_{2 j-1}[j]= & \frac{1}{(2 j-1) !}(\cos (\theta / 2))(i \sin (\theta / 2))^{2 j-1}, \\
C_{2 j-2}[j]= & \frac{1}{(2 j-2) !}(i \sin (\theta / 2))^{2 j-2}\left(1+\frac{1}{3}(j-1) \sin ^{2}(\theta / 2)\right), \\
C_{2 j-3}[j]= & \frac{1}{(2 j-3) !}(\cos (\theta / 2))(i \sin (\theta / 2))^{2 j-3}\left(1+\frac{1}{3} j \sin ^{2}(\theta / 2)\right), \\
C_{2 j-4}[j]= & \frac{1}{(2 j-4) !}(i \sin (\theta / 2))^{2 j-4} \\
& \times\left(1+\frac{1}{3}(j-2) \sin ^{2}(\theta / 2)+\frac{1}{90}(5 j+1)(j-2) \sin ^{4}(\theta / 2)\right), \\
C_{2 j-5}[j]= & \frac{1}{(2 j-5) !}(\cos (\theta / 2))(i \sin (\theta / 2))^{2 j-5} \\
& \times\left(1+\frac{1}{3}(j-1) \sin ^{2}(\theta / 2)+\frac{1}{90}(5 j+1) j \sin ^{4}(\theta / 2)\right),
\end{aligned}
$$

etc.

In general then, for any integer $n$, so long as $n \leq j$,

$$
\begin{aligned}
& C_{2 j-2 n+1}[j]=\frac{(i \sin (\theta / 2))^{2 j-2 n+1} \cos (\theta / 2)}{(2 j-2 n+1) !} \operatorname{Trunc}_{n-1}\left[\left.\frac{1}{\sqrt{1-x}}\left(\frac{\arcsin \sqrt{x}}{\sqrt{x}}\right)^{2 j-2 n+1}\right|_{x=\sin ^{2}(\theta / 2)},(6)\right. \\
& C_{2 j-2 n}[j]=\frac{(i \sin (\theta / 2))^{2 j-2 n}}{(2 j-2 n) !} \operatorname{Trunc}_{n}\left[\left.\left(\frac{\arcsin \sqrt{x}}{\sqrt{x}}\right)^{2 j-2 n}\right|_{x=\sin ^{2}(\theta / 2)},\right.
\end{aligned}
$$

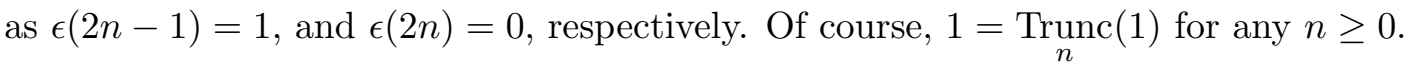

Note from these expressions that half of the $C$ s for a given $j$ are related by a simple derivative recursive condition between contiguous coefficients,

$$
i C_{2(j-n)-1}[j]=2 \frac{d}{d \theta} C_{2(j-n)}[j] \quad \text { for } \quad n \geq 0,
$$

that is, derivation of the $\epsilon=0$ terms yields the contiguous $\epsilon=1$ terms. 
The sequence of coefficients terminates at $C_{0}[j]$, of course, which, on the one hand, for integer $j$ requires $n=j$ in (7), giving $C_{0}[n]=1$.

But, on the other hand, for half integer $j$, it requires $n=j+1 / 2$ in (6), giving

$$
C_{0}\left[\frac{2 n-1}{2}\right]=\left.\cos (\theta / 2) \operatorname{Trunc}_{n-1}\left(\frac{1}{\sqrt{1-x}}\right)\right|_{x=\sin ^{2}(\theta / 2)} .
$$

Moreover, for half-integral spins, derivation of this leading, $\epsilon=1$ term, yields the last, $\epsilon=0$ term,

$$
2 i \frac{d}{d \theta} C_{0}[j]=C_{2 j}[j]\left(\frac{(2 j !)}{(j-1 / 2) ! 2^{j-1 / 2}}\right)^{2}(-1)^{j-1 / 2} .
$$

This is the only distinction between integer and semi-integer spins in this analytic top-down approach, although these two $C_{0} \mathrm{~s}$ can be compacted to a single expression upon using the familiar discrete Bose/Fermi index, or spin discriminant, a binary variable,

$$
\epsilon(j)=\left(1-(-1)^{2 j}\right) / 2=2(j-\lfloor j\rfloor)= \begin{cases}0 & \text { if } j \text { is integer } \\ 1 & \text { if } j \text { is half-integer }\end{cases}
$$

By use of the same discriminant, the coefficients can be written in a common form for either an even or an odd number of steps down from $2 j$. Thus for any descending order $m \in$ $[0,1, \ldots, 2 j]$, we have $\epsilon(m / 2)=1$ if $m$ is an odd integer and $\epsilon(m / 2)=0$ if $m$ is an even integer, so that

$$
C_{2 j-m}[j]=\frac{(\cos (\theta / 2))^{\epsilon(m / 2)}(i \sin (\theta / 2))^{2 j-m}}{(2 j-m) !} \operatorname{Trunc}_{\left\lfloor\frac{m}{2}\right\rfloor}\left[\left(\frac{1}{\sqrt{1-x}}\right)^{\epsilon(m / 2)}\left(\frac{\arcsin \sqrt{x}}{\sqrt{x}}\right)^{2 j-m}\right],
$$

where $x \equiv \sin ^{2}(\theta / 2)$ and $\lfloor\ldots\rfloor$ is the floor function, with $n=\left\lfloor\frac{2 n}{2}\right\rfloor=\left\lfloor\frac{2 n+1}{2}\right\rfloor$. The interchanged roles of ascending and descending indices is evident upon comparison with the equivalent formula (1).

\section{Generating functions}

In some contrast to the previous section, derivatives of $\epsilon=1$ coefficients are related to other coefficients, but, in general, of lower spins $j$ and of both $\epsilon=0$ and $\epsilon=1$.

For instance, for integer $j$ and integer $n$, and for $C_{0}[0] \equiv 1, C_{1}[0] \equiv 0$,

$$
\begin{aligned}
\left(2 \frac{d}{d \theta}+\tan (\theta / 2)\right) C_{2 n+1}[j]= & \sum_{m=0}^{j-n-1}\left(i \cos ^{2}(\theta / 2) \sin ^{2 m}(\theta / 2) C_{2 n}[j-m-1]\right. \\
& \left.+\cos (\theta / 2) \sin ^{2 m+1}(\theta / 2) C_{2 n+1}[j-m-1]\right) .
\end{aligned}
$$

For half-integer $j$, likewise,

$$
\begin{aligned}
\left(2 \frac{d}{d \theta}+\tan (\theta / 2)\right) C_{2 n}[j]= & \sum_{m=0}^{j-n-3 / 2}\left(i \cos ^{2}(\theta / 2) \sin ^{2 m}(\theta / 2) C_{2 n-1}[j-m-1]\right. \\
& \left.+\cos (\theta / 2) \sin ^{2 m+1}(\theta / 2) C_{2 n}[j-m-1]\right) .
\end{aligned}
$$


The top-down coefficients (6), (7) may be encoded systematically in a pair of Generating functions linking all different spins $j$, and reliant on the incomplete Gamma function,

$$
\Gamma(n+1, z) \equiv \int_{z}^{\infty} e^{-t} t^{n} d t, \quad \Gamma(n+1,0)=\Gamma(n+1)=n !,
$$

albeit with operator arguments.

Letting $j=m / 2$ and summing over $m=0,1,2, \ldots, \infty$ to cover all spins, both integer and half integer, the master generating functions are

$$
\begin{aligned}
G_{2 n}(t, x) & \equiv \sum_{m=0}^{\infty} t^{m} C_{m-2 n}[m / 2]=\sum_{m=2 n}^{\infty} t^{m} C_{m-2 n}[m / 2] \\
& =t^{2 n}\left[\left.\sum_{r=0}^{n} \frac{x^{r}}{r !} \frac{d^{r}}{d y^{r}} \exp \left(\frac{t \sqrt{x} \arcsin \sqrt{y}}{\sqrt{y}}\right)\right|_{y=0}\right. \\
& =\frac{1}{n !} t^{2 n}\left[\left.\Gamma\left(n+1, x \frac{d}{d y}\right) \exp \left(\frac{t \sqrt{x} \arcsin \sqrt{y+x}}{\sqrt{y+x}}\right)\right|_{y=0}\right.
\end{aligned}
$$

and

$$
\begin{aligned}
G_{2 n+1}(t, x) & \equiv \sum_{m=0}^{\infty} t^{m} C_{m-2 n+1}[m / 2]=\sum_{m=2 n-1}^{\infty} t^{m} C_{m-2 n+1}[m / 2] \\
& =t^{2 n-1} \sqrt{1-x}\left[\left.\sum_{r=0}^{n-1} \frac{x^{r}}{r !} \frac{d^{r}}{d y^{r}}\left(\frac{1}{\sqrt{1-y}} \exp \left(\frac{t \sqrt{x} \arcsin \sqrt{y}}{\sqrt{y}}\right)\right)\right|_{y=0}\right. \\
& =\frac{1}{n !} t^{2 n-1} \sqrt{1-x}\left[\left.\Gamma\left(n+1, x \frac{d}{d y}\right)\left(\frac{1}{\sqrt{1-x-y}} \exp \left(\frac{t \sqrt{x} \arcsin \sqrt{y+x}}{\sqrt{y+x}}\right)\right)\right|_{y=0} .\right.
\end{aligned}
$$

Thus, for instance,

$$
G_{0}=\sum_{m=0}^{\infty} t^{m} C_{m}[m / 2]=\sum_{m=0}^{\infty} \frac{t^{m}}{m !}(i \sin (\theta / 2))^{m}=e^{i t \sin (\theta / 2)},
$$

yields (4) for each $j=m / 2$;

$$
\begin{aligned}
G_{1} & =\sum_{m=0}^{\infty} t^{m} C_{m-1}[m / 2]=\sum_{m=1}^{\infty} t^{m} C_{m-1}[m / 2] \\
& =\sum_{m=1}^{\infty} \frac{t^{m}}{(m-1) !}(\cos (\theta / 2))(i \sin (\theta / 2))^{m-1}=(\cos (\theta / 2)) t e^{i t \sin (\theta / 2)}
\end{aligned}
$$

yields (5) for each $j=m / 2$;

$$
\begin{aligned}
G_{2} & =\sum_{m=0}^{\infty} t^{m} C_{m-2}[m / 2]=\sum_{m=2}^{\infty} t^{m} C_{m-2}[m / 2] \\
& =\sum_{m=2}^{\infty} \frac{t^{m}}{(m-2) !}(i \sin (\theta / 2))^{m-2}\left(1+\frac{1}{3}\left(\frac{m}{2}-1\right) \sin ^{2}(\theta / 2)\right) \\
& =t^{2} \text { KummerM }\left(\frac{\sin ^{2}(\theta / 2)+6}{\sin ^{2}(\theta / 2)}, \frac{6}{\sin ^{2}(\theta / 2)}, i t \sin (\theta / 2)\right)
\end{aligned}
$$

etc., involving hypergeometric functions. 


\section{Specification of the series}

Calculation of the rotation matrices as spin polynomials can be efficiently carried out in specific cases using either Lagrange-Sylvester expansions, or the Cayley-Hamilton theorem. These two methods are tied together by Vandermonde matrix algebra [1]. The expressions for general spin $j$ given in the first section of this paper can be established using these methods.

Consider functions of an $N \times N$ diagonalizable matrix $\mathbb{M}$ with non-degenerate eigenvalues $\lambda_{i}$, $i=1, \ldots, N$. On the span of the eigenvectors, there is an obviously correct Lagrange formula, as extended to matrices by Sylvester,

$$
f(\mathbb{M})=\sum_{i=1}^{N} f\left(\lambda_{i}\right) \mathbb{P}_{i},
$$

where the projection operators - the so-called Frobenius covariants - are given by products,

$$
\mathbb{P}_{i}=\prod_{\substack{j=1 \\ j \neq i}}^{N} \frac{\mathbb{M}-\lambda_{j}}{\lambda_{i}-\lambda_{j}}
$$

From expanding each such product it is evident that any $f(\mathbb{M})$ reduces to a polynomial of order $N-1$ in powers of $\mathbb{M}$,

$$
f(\mathbb{M})=\sum_{m=0}^{N-1} C_{m}[f] \mathbb{M}^{m},
$$

and the function-dependent coefficients can be expressed in terms of the eigenvalues of $\mathbb{M}$ by expanding the projection operators (9) as polynomials in $\mathbb{M}$.

The condition that no two eigenvalues be the same in (9) can be efficiently implemented using Vandermonde matrix methods. From the polynomial (10) acting on eigenvector $\left|\lambda_{k}\right\rangle$ we obtain

$$
f\left(\lambda_{k}\right)=\sum_{m=0}^{N-1} C_{m}[f]\left(\lambda_{k}\right)^{m} .
$$

The action on the full set of $N$ eigenvectors therefore gives an $N \times N$ matrix equation,

$$
\left(\begin{array}{c}
f\left(\lambda_{1}\right) \\
f\left(\lambda_{2}\right) \\
\vdots \\
f\left(\lambda_{N}\right)
\end{array}\right)=V[\mathbb{M}]\left(\begin{array}{c}
C_{0}[f] \\
C_{1}[f] \\
\vdots \\
C_{N-1}[f]
\end{array}\right)
$$

where the $N \times N$ Vandermonde matrix of $\mathbb{M}$ eigenvalues is

$$
V[\mathbb{M}]=\left(\begin{array}{ccccc}
1 & \lambda_{1} & \lambda_{1}^{2} & \ldots & \lambda_{1}^{N-1} \\
1 & \lambda_{2} & \lambda_{2}^{2} & \ldots & \lambda_{2}^{N-1} \\
\vdots & \vdots & \vdots & \ddots & \vdots \\
1 & \lambda_{N} & \lambda_{N}^{2} & \ldots & \lambda_{N}^{N-1}
\end{array}\right)
$$

As is well-known, for nondegenerate eigenvalues, $V$ is nonsingular [6]. Thus, one may obtain the coefficients $C_{k}[f]$ in the expansion (10) by merely inverting $V$ in (11), into the inverse 
relation,

$$
\left(\begin{array}{c}
C_{0}[f] \\
C_{1}[f] \\
\vdots \\
C_{N-1}[f]
\end{array}\right)=V^{-1}[\mathbb{M}]\left(\begin{array}{c}
f\left(\lambda_{1}\right) \\
f\left(\lambda_{2}\right) \\
\vdots \\
f\left(\lambda_{N}\right)
\end{array}\right)
$$

although one should note that, by our conventions, the row index $m$ runs from 0 to $N-1$, while the column index $i$ appears more familiar, running from 1 to $N$.

For the problem at hand, we are interested in the case where the matrix is $\mathbb{M}=2 \hat{\boldsymbol{n}} \cdot \boldsymbol{J}$. Thus for spin $j$ we have a matrix $\mathbb{M}$ whose dimension is $N=2 j+1$ and whose eigenvalues are $\lambda_{k}=2(j+1-k)$, ordered so that $\left\{\lambda_{1}, \lambda_{2}, \ldots, \lambda_{N}\right\}=\{2 j, 2 j-2, \ldots,-2 j\}$. The Vandermonde matrix for spin $j$ is then $(2 j+1) \times(2 j+1)$ as given by

$$
V[j]=\left(\begin{array}{ccccc}
1 & 2 j & (2 j)^{2} & \cdots & (2 j)^{2 j} \\
1 & 2 j-2 & (2 j-2)^{2} & \cdots & (2 j-2)^{2 j} \\
\vdots & \vdots & \vdots & \ddots & \vdots \\
1 & -2 j & (-2 j)^{2} & \cdots & (-2 j)^{2 j}
\end{array}\right)
$$

Note here that $\operatorname{det} V[j] \neq 0$, hence $V^{-1}[j]$ exists. In fact,

$$
\operatorname{det} V[j]=(-1)^{\left\lfloor\frac{2 j+1}{2}\right\rfloor} \prod_{\text {prime } p \leq 2 j} p^{m_{p}(j)},
$$

where $m_{p}(j)$ is the multiplicity of the prime $p$ that occurs in the factorization of $\operatorname{det} V[j]$. Explicitly,

$$
\begin{aligned}
& m_{2}(j)=\sum_{k=1}^{2 j} \sum_{m=0}^{\lfloor\ln k / \ln 2\rfloor}\left\lfloor\frac{k}{2^{m}}\right\rfloor, \quad \text { and } \\
& m_{p}(j)+j(2 j+1)=\sum_{k=1}^{2 j} \sum_{m=0}^{\lfloor\ln k / \ln p\rfloor}\left\lfloor\frac{k}{p^{m}}\right\rfloor, \quad \text { for } \quad p \geq 3 .
\end{aligned}
$$

Note that a given prime $p>2$ first appears in the factorization of the determinant for fermionic spin $j=p / 2$, and subsequently appears as a factor in $\operatorname{det} V[j]$ for all higher spins.

Returning to the problem at hand, we are interested in the exponential function, $\exp (\alpha \mathbb{M})$, so by $(10)$,

$$
\exp (2 \alpha \hat{\boldsymbol{n}} \cdot \boldsymbol{J})=\sum_{m=0}^{2 j} C_{m}[j](2 \hat{\boldsymbol{n}} \cdot \boldsymbol{J})^{m},
$$

where each $j$ dependent coefficient $C_{m}[j]$ is also implicitly a function of $\alpha$, to be determined. Of course, for rotations $\alpha=i \theta / 2$. These functions are obtained by (12)) specialized to this case, namely,

$$
\left(\begin{array}{c}
C_{0}[j] \\
C_{1}[j] \\
\vdots \\
C_{2 j}[j]
\end{array}\right)=V^{-1}[j]\left(\begin{array}{c}
e^{2 j \alpha} \\
e^{(2 j-2) \alpha} \\
\vdots \\
e^{-2 j \alpha}
\end{array}\right)
$$


Thus the $C_{k} \mathrm{~s}$ are linear combinations of the $2 j+1$ exponentials shown. It remains only to compute the inverse of the Vandermonde matrix for spin $j$, and to express the results for the $C_{k} \mathrm{~s}$ in a compact form.

One immediate result involves $C_{0}$ for integer spins: Given our ordering of the eigenvalues, the first row of $V^{-1}[j$ integer] consists entirely of zeroes, except for a 1 in the middle column (i.e. the $(j+1)$ st) corresponding to the obvious fact that the middle row (again the $(j+1)$ st) of $V[j$ integer $]$ has a 1 in the first column and zeroes in all subsequent columns (since $0^{k}=0$ for all positive $k$ ). But the middle entry in the column of exponentials on the r.h.s. of (14) is just $e^{0}=1$. Therefore $C_{0}=1$ for all integer $j$.

All the coefficients $C_{k}$ satisfy first-order differential relations. Half of these relations are simply of the form $C_{n-1}=d C_{n} / d \alpha$ while the other half are more involved. Above, we presented an interesting form for the more elaborate cases, where a first-order equation mixed spin $j$ coefficients with all lower spins of the same type, either all integer or all semi-integer. Here, we take a different approach, so that different spin coefficients are not mixed ab initio. Nonetheless, in our labeling of the coefficients as $C_{m}[j]$, we have chosen to keep track of the spin $j$ in addition to the power of $\hat{\boldsymbol{n}} \cdot \boldsymbol{J}$, as in the previous section, because the form of some key results can be used to obtain recursion relations relating different spins.

First consider the simplest results which apply to half the coefficients. For clarity, we give separately the results for the integer and semi-integer cases. For integer $j$,

$$
\frac{d}{d \alpha} C_{2 k}[j]=C_{2 k-1}[j]
$$

where, for spin zero, $C_{0}[0] \equiv 1$, and $C_{1}[0] \equiv 0$. For semi-integer $j$,

$$
\frac{d}{d \alpha} C_{2 k+1}[j]=C_{2 k}[j] \text {. }
$$

We now describe an approach which applies to all other cases just as well. Differentiating the coefficients in the expansion of the exponential (13) gives on the one hand,

$$
\frac{d}{d \alpha} \exp (2 \alpha \hat{\boldsymbol{n}} \cdot \boldsymbol{J})=\sum_{m=0}^{2 j} \frac{d}{d \alpha} C_{m}[j](2 \hat{\boldsymbol{n}} \cdot \boldsymbol{J})^{m},
$$

while, on the other hand, just from differentiating the exponential itself, it follows that

$$
\begin{aligned}
\frac{d}{d \alpha} \exp (2 \alpha \hat{\boldsymbol{n}} \cdot \boldsymbol{J}) & =2 \hat{\boldsymbol{n}} \cdot \boldsymbol{J} \exp (2 \alpha \hat{\boldsymbol{n}} \cdot \boldsymbol{J}) \\
& =\sum_{m=1}^{2 j} C_{m-1}[j](2 \hat{\boldsymbol{n}} \cdot \boldsymbol{J})^{m}+C_{2 j}[j](2 \hat{\boldsymbol{n}} \cdot \boldsymbol{J})^{2 j+1} .
\end{aligned}
$$

We obtain first-order differential relations for the coefficients by equating the two expressions (18) and (17). To make further progress, it is necessary to resolve $(2 \hat{\boldsymbol{n}} \cdot \boldsymbol{J})^{2 j+1}$ as a series in lower powers of $2 \hat{\boldsymbol{n}} \cdot \boldsymbol{J}$.

This resolution can be achieved by applying the Cayley-Hamilton theorem to the spin $j>0$ matrices. The final result is straightforward to obtain:

$$
(2 \hat{\boldsymbol{n}} \cdot \boldsymbol{J})^{2 j+1}=\sum_{m=0}^{2 j} A_{m}[j](2 \hat{\boldsymbol{n}} \cdot \boldsymbol{J})^{m}
$$

where the coefficients are explicitly encoded in the following polynomial, valid for either integer or semi-integer spin:

$$
p_{j}(x) \equiv x^{2 j+1}-\frac{1}{x^{2 j+1}} \prod_{m=0}^{2 j}\left(x^{2}-\left(\frac{1+(-1)^{m+2 j}}{2}\right) m^{2}\right)=\sum_{m=0}^{2 j} A_{m}[j] x^{m} .
$$


The resolution of $(2 \hat{\boldsymbol{n}} \cdot \boldsymbol{J})^{2 j+1}$ may also be reduced to a Vandermonde matrix equation when acting with (19) on the eigenvectors of $2 \hat{\boldsymbol{n}} \cdot \boldsymbol{J}$, and leads again to the results encoded in the $p_{j}(x)$ given in $(20)$.

To connect with existing mathematical literature, it is worthwhile to note the coefficients for semi-integer spins may be appropriately called fermionic central factorial numbers, i.e. "fcfn", but they are actually known in the literature as just central factorial numbers [2] ${ }^{1}$. Similarly, the $A_{m}[j]$ for integer spins may be called the bosonic central factorial numbers, i.e. "bcfn", but they are known in the mathematics literature as the scaled central factorial numbers ${ }^{2}$. By interlacing the rows and columns of the fcfn and bcfn matrices, the coefficients for both integer and semi-integer cases can be expressed in a unified manner in terms of a single matrix, the result being effectively a replication of $(20)$.

Combining (18), (17), and (19) leads to

$$
\frac{d C_{m}[j]}{d \alpha}=C_{m-1}[j]+C_{2 j}[j] A_{m}[j] \quad \text { for } \quad m=0, \ldots, 2 j
$$

with the convention $C_{-1}[j]=0$, which immediately shows two noteworthy features: The simplicity of $d C_{0}[j] / d \alpha$ and $d C_{1}[j] / d \alpha$ for any $j$. The coefficients of the lowest and highest powers of $(2 \hat{\boldsymbol{n}} \cdot \boldsymbol{J})$ are linked by differentiation. For example,

$$
\frac{d}{d \alpha} C_{0}[j]=(-1)^{\lfloor j\rfloor}[(2 j) ! !]^{2} C_{2 j}[j] \quad \text { for } \quad j \text { semi-integer. }
$$

Similarly, since $C_{0}[j$ integer $]=1$,

$$
\frac{d C_{1}[j]}{d \alpha}=1+(-1)^{j-1}[(2 j) ! !]^{2} C_{2 j}[j] \quad \text { for } j \text { integer. }
$$

Symmetry under the reflection map $\hat{\boldsymbol{n}} \cdot \boldsymbol{J} \longmapsto-\hat{\boldsymbol{n}} \cdot \boldsymbol{J}$ implies only odd integer $m$ contribute on the r.h.s. of (19) for integer $j$, i.e. $A_{2 k}[j$ integer $]=0$, and only even integer $m$ contribute for half-integer $j$, i.e. $A_{2 k+1}[j$ half-integer $]=0$. This suffices to establish (15) and (16), since (21) reduces to those simple results for $m=2 k$ and integer $j$, and for $m=2 k+1$ and semi-integer $j$, respectively.

The remaining cases are less trivial, as given by

$$
\begin{aligned}
& \frac{d C_{2 k+1}[j]}{d \alpha}=C_{2 k}[j]+\frac{1}{(2 j) !}(\sinh \alpha)^{2 j} A_{2 k+1}[j] \quad \text { for integer } j, \\
& \frac{d C_{2 k}[j]}{d \alpha}=C_{2 k-1}[j]+\frac{1}{(2 j) !}(\sinh \alpha)^{2 j} A_{2 k}[j] \quad \text { for semi-integer } j,
\end{aligned}
$$

where we have used the fact that

$$
C_{2 j}[j]=\frac{1}{(2 j) !}(\sinh \alpha)^{2 j},
$$

manifest by inspection of the highest-order term in the Lagrange-Sylvester expansions, (8)-(10).

Given the coefficients $A_{m}[j]$ then, as provided by (20), the coefficients $C_{m}[j]$ are computed successively from the lowest to the highest values of $m$ by integration of (22) and (23), or from highest to lowest values of $m$, by carrying out the differentiations in those two equations. The results of this algorithm were given at the beginning of this paper. (For a distinctly different, albeit presumably equivalent algorithm, see [12].)

\footnotetext{
${ }^{1}$ These are presented as triangular matrices in http://oeis.org/A008956.

${ }^{2}$ These are presented as triangular matrices in http://oeis.org/A182867.
} 
So far in this section, only a single spin $j$ is involved in each relation. Nevertheless, the explicit form for the polynomials in (20)) displays how the $p_{j}$ obey recursion relations that relate different spins, namely,

$$
p_{j+1}(x)=(2 j+2)^{2} x^{2 j+1}+\left(x^{2}-(2 j+2)^{2}\right) p_{j}(x) .
$$

This permits relating $A_{k}[j+1]$ and $A_{m}[j]$, and hence relating the derivatives of the $C_{m} \mathrm{~s}$ for different spins. This, in turn, opens a route to arriving at all the first-order relations given in the previous section.

The explicit forms given here for the $C_{m} \mathrm{~s}$ are, in fact, just the solutions of those firstorder relations. In this way, a rigorous proof of (1) may be obtained, as a reader may readily verify - and illustrate for a low $j$. Fortunately, it is not actually necessary to go through the straightforward but tedious details of such a proof here: Since this paper appeared on the arXiv, a simplified derivation, based on (21) and closed-form expressions for $A_{m}[j]$ in terms of central factorial numbers has also appeared [3].

\section{Concluding remarks}

Rotation matrices for all representations $j$ have been expressed as $2 j$-order polynomials in the corresponding spin matrices, equation (1). A plethora of applications [12] of these elementary results can be easily imagined, across the entire spectrum of quantum mechanical systems, including problems in atomic, condensed matter, nuclear, and elementary particle physics $[7,16]$.

Such applications would range from the most straightforward, e.g. using the formula (1) to compute Wigner's $d$ functions starting from a specific representation of $J_{y}$, to the more esoteric, e.g., investigation of the large $j$ asymptotics in various contrived matrix models.

Specifically, consider

$$
\begin{aligned}
\operatorname{det}(\lambda \mathbf{1}-2 \hat{\boldsymbol{n}} \cdot \boldsymbol{J}) & =\frac{2^{2 j+1}}{\pi} \Gamma\left(1+j+\frac{1}{2} \lambda\right) \Gamma\left(1+j-\frac{1}{2} \lambda\right) \sin \pi\left(\frac{1}{2} \lambda-j\right) \\
& \sim \underset{j \rightarrow \infty}{\sim} \frac{1}{\pi} 2^{2 j+1}(j !)^{2} \sin \pi\left(\frac{1}{2} \lambda-j\right) .
\end{aligned}
$$

The two $\Gamma$ factors have poles that terminate the infinite sequence of $\sin \pi\left(\frac{1}{2} \lambda-j\right)$ zeroes both above and below, so that setting the r.h.s. of (24) to zero indeed reproduces the finite set of eigenvalues: $2 j, 2 j-2, \ldots,-2 j+2,-2 j$, as it should.

Alternatively, with the benefit of Stirling's formula, (24) becomes

$$
\prod_{n=0}^{2 j}(\lambda-2(j-n)) \underset{j \rightarrow \infty}{\sim} 2 e^{-2 j}(2 j)^{2 j+1} \begin{cases}(-1)^{j} \sin \pi\left(\frac{1}{2} \lambda\right) & \text { for } j \text { integer, } \\ (-1)^{j+\frac{1}{2}} \cos \pi\left(\frac{1}{2} \lambda\right) & \text { for } j \text { semi-integer }\end{cases}
$$

and thereby provides a route to reach Euler's infinite product representation of the sine and cosine functions.

In particular, as illustrated in Section 3, the large $j$ limit warrants some additional mention here, since it is intriguingly intuitive even in the completely quantum/noncommutative framework of this work. In the limit $j \rightarrow \infty$, the coefficients of the various powers, $(2 i \hat{\boldsymbol{n}} \cdot \boldsymbol{J})^{n} / n$ ! in the expansion of the rotation group element become quasi-classical: if $\hat{\boldsymbol{n}} \cdot \boldsymbol{J}$ were not a matrix, but a scalar instead, these coefficients in the exponential series would be just simple monomials, $(\theta / 2)^{n}$.

Remarkably, the coefficients presented in (1) reduce, in this limit, to elegant trigonometric series [9] for the periodicized monomials - exactly! -

$$
\lim _{j \rightarrow \infty} c_{k}(\theta) \sin ^{k}(\theta / 2)=(-1)^{\left(1+\left\lfloor\frac{\theta}{2 \pi}-\frac{1}{2}\right\rfloor\right) \epsilon(j)}\left(\frac{1}{2}\left(\theta-2 \pi-2 \pi\left\lfloor\frac{\theta}{2 \pi}-\frac{1}{2}\right\rfloor\right)\right)^{k} .
$$


Indeed, for finite $j$, the coefficients are nothing but the Taylor polynomial truncations of these same trigonometric series. But even in the limit of large $j$ there is always a clear distinction between the integer and semi-integer spin cases, as given by the fermionic sign flip in this equation for $\pi<|\theta|<2 \pi$. This is illustrated in the appendix.

This appears as an elegant connection between the rotation group and Fourier analysis, moreover underlain by subtle combinatorial identities involving the central factorial numbers, as we have explained in the previous section. Thus, the results presented evoke an intriguing set of linkages between group theory, combinatorics, and analysis.

Extensions to $\mathrm{SO}(4)$ and $\mathrm{SU}_{q}(2)$ are direct. In fact, the $\mathrm{SO}(4)$ case would be of some physical interest in superintegrable models involving extensions of the Hermann-Bernoulli-LaplaceHamilton-Gibbs-Runge-Lenz-Pauli vector to cases of arbitrary spin [8]. Perhaps the results here can be of use in the analysis of such models.

\section{A Appendix. Periodicized monomials}

The remarks in the text about the behavior of the coefficients in the large $j$ limit are illustrated here for the coefficients of the lowest three powers of the generators with $-2 \pi \leq \theta \leq 2 \pi$, for spins $j=137 / 2$ (shown in blue) and $j=69$ (shown in purple). Even in the limit of large $j$ there is always a clear distinction between the integer and semi-integer spin cases, as given by a fermionic sign flip for $\pi<|\theta|<2 \pi$.

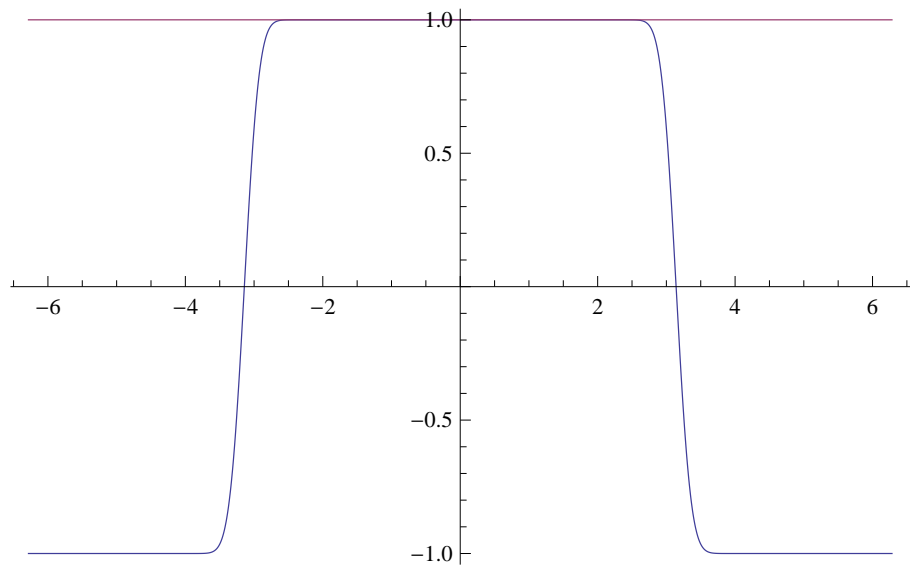

$c_{0}$ versus $\theta$

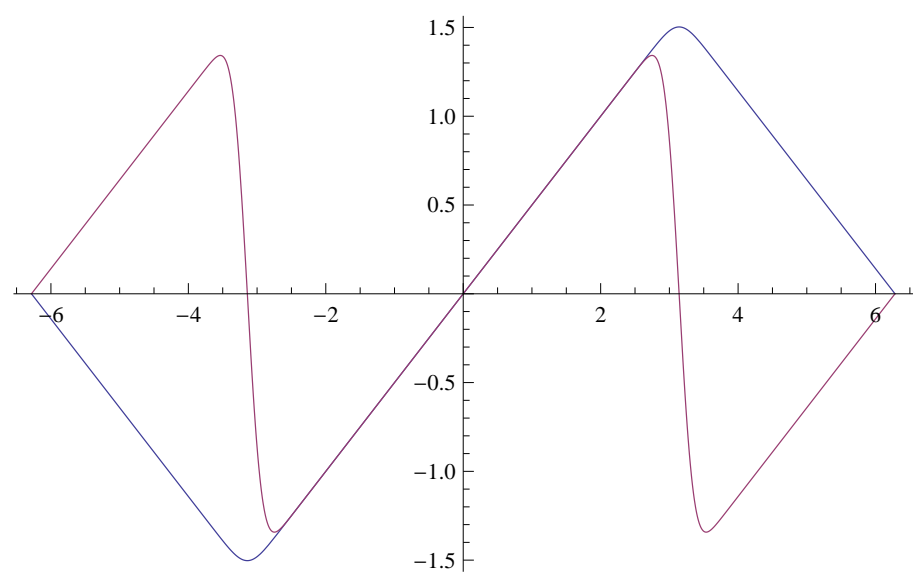

$c_{1} \sin (\theta / 2)$ versus $\theta$ 


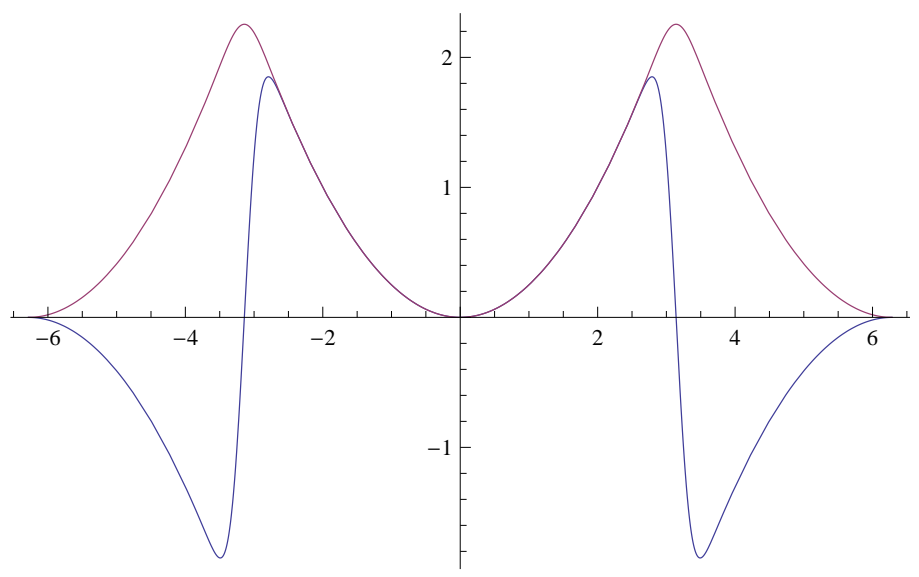

$c_{2} \sin ^{2}(\theta / 2)$ versus $\theta$

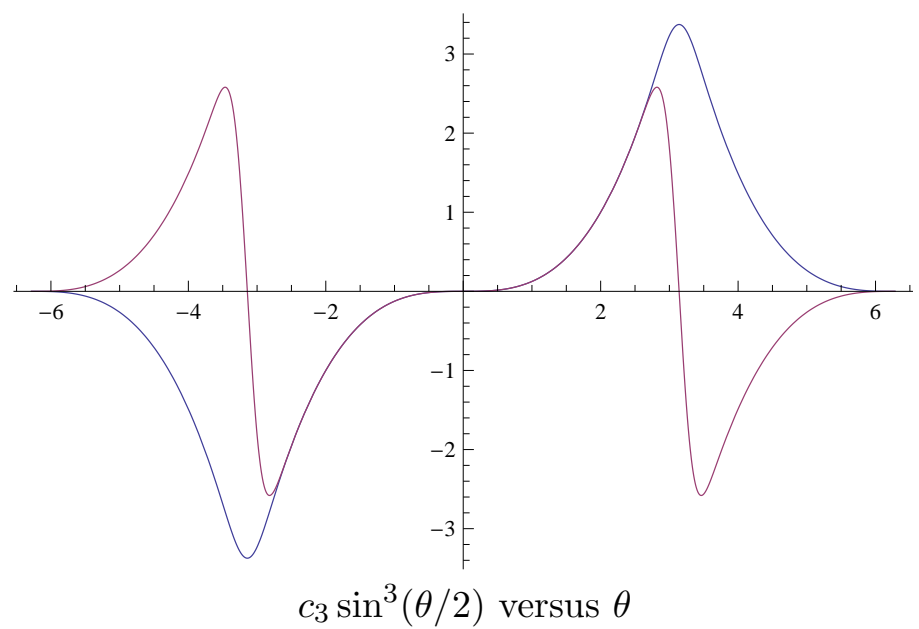

These plots should be compared to the exact discontinuous functions obtained in the limit, as given by the last formula in the text. Note the striking absence of Gibbs-Wilbraham phenomena [9] in these figures. Each term in the Taylor polynomials Trunc of (2) is positive semi-definite.

\section{Acknowledgements}

The submitted manuscript has been created by UChicago Argonne, LLC, Operator of Argonne National Laboratory (Argonne). Argonne, a U.S. Department of Energy Office of Science laboratory, is operated under Contract No. DE-AC02-06CH11357. The U.S. Government retains for itself, and others acting on its behalf, a paid-up nonexclusive, irrevocable worldwide license in said article to reproduce, prepare derivative works, distribute copies to the public, and perform publicly and display publicly, by or on behalf of the Government. It was also supported in part by NSF Award PHY-1214521. TLC was also supported in part by a University of Miami Cooper Fellowship. S. Dowker is thanked for bringing ref [12], and whence [5], to our attention. An anonymous referee is especially thanked for bringing [14] and more importantly [13] to our attention.

\section{References}

[1] Atiyah M.F., Macdonald I.G., Introduction to commutative algebra, Addison-Wesley Publishing Co., Reading, Mass. - London - Don Mills, Ont., 1969.

[2] Butzer P.L., Schmidt M., Stark E.L., Vogt L., Central factorial numbers; their main properties and some applications, Numer. Funct. Anal. Optim. 10 (1989), 419-488. 
[3] Curtright T.L., Van Kortryk T.S., On rotations as spin matrix polynomials, arXiv:1408.0767.

[4] Euler L., Problema algebraicum ob affectiones prorsus singulares memorabile, Commentatio 407 Indicis Enestoemiani, Novi Comm. Acad. Sci. Petropolitanae 15 (1770), 75-106.

[5] Lehrer-Ilamed Y., On the direct calculations of the representations of the three-dimensional pure rotation group, Math. Proc. Cambridge Philos. Soc. 60 (1964), 61-66.

[6] Macon N., Spitzbart A., Inverses of Vandermonde matrices, Amer. Math. Monthly 65 (1958), 95-100.

[7] Nelson T.J., Spin-matrix polynomials and the Veneziano formula, Phys. Rev. 184 (1969), $1954-1955$.

[8] Nikitin A.G., Laplace-Runge-Lenz vector for arbitrary spin, J. Math. Phys. 54 (2013), 123506, 14 pages, arXiv:1308.4279.

[9] Pinsky M.A., Introduction to Fourier analysis and wavelets, Graduate Studies in Mathematics, Vol. 102, Amer. Math. Soc., Providence, RI, 2009.

[10] Rodrigues O., Des lois géometriques qui regissent les déplacements d' un systéme solide dans l' espace, et de la variation des coordonnées provenant de ces déplacement considerées indépendent des causes qui peuvent les produire, J. Math. Pures Appl. 5 (1840), 380-400.

[11] Schwinger J., On angular momentum, report NYO-3071, Harvard University, Nuclear Development Associates, Inc. (US), 1952.

[12] Torruella A.J., Representations of the three-dimensional rotation group by the direct method, J. Math. Phys. 16 (1975), 1637-1642.

[13] van Wageningen R., Explicit polynomial expressions for finite rotation operators, Nuclear Phys. 60 (1964), $250-263$.

[14] Weber T.A., Williams S.A., Spin-matrix polynomials and the rotation operator for arbitrary spin, J. Math. Phys. 6 (1965), 1980-1983.

[15] Wigner E.P., Group theory and its application to the quantum mechanics of atomic spectra, Pure and Applied Physics, Vol. 5, Academic Press, New York - London, 1959.

[16] Williams S.A., Draayer J.P., Weber T.A., Spin-matrix polynomial development of the Hamiltonian for a free particle of arbitrary spin and mass, Phys. Rev. 152 (1966), 1207-1212.

[17] Zygmund A., Trigonometric series: Vols. I, II, Cambridge University Press, London - New York, 1968. 